\title{
Testing for the Monotone Likelihood Ratio Assumption
}

\author{
Jutta Roosen and David A. Hennessy
}

Working Paper 03-WP 325

February 2003

\section{Center for Agricultural and Rural Development lowa State University \\ Ames, lowa 50011-1070 \\ www.card.iastate.edu}

Jutta Roosen is an assistant professor in the Unit of Agricultural Economics and invited member of CORE, both at the University of Louvain, Belgium. David Hennessy is a professor in the Center for Agricultural and Rural Development and Department of Economics at lowa State University.

The authors thank, without implication, Brent Hueth for conversations on empirical contract design issues and Jay Shogren for providing the auction data as well as for insightful discussions on value affiliation.

This publication is available online on the CARD website: www.card.iastate.edu. Permission is granted to reproduce this information with appropriate attribution to the authors and the Center for Agricultural and Rural Development, lowa State University, Ames, lowa 50011-1070.

For questions or comments about the contents of this paper, please contact Jutta Roosen, University of Louvain, Unit of Agricultural Economics, Place Croix du Sud 2/15, B-1348 Louvainla-Neuve, Belgium; Ph: +32 (0)10 4787 07; Fax: +32 (0)10 4736 75; E-mail:

roosen@ecru.ucl.ac.be. Contact David Hennessy at 578C Heady Hall, lowa State University, Ames, IA 50011-1070; Ph: 515-294-6171; Fax: 515-294-6336; E-mail: hennessy@iastate.edu.

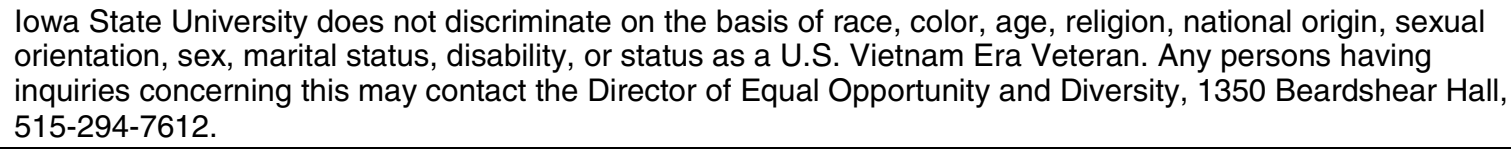
orientation, sex, marital status, disability, or status as a U.S. Vietnam Era Veteran. Any persons having inquiries concerning this may contact the Director of Equal Opportunity and Diversity, 1350 Beardshear Hall, 515-294-7612. 


\begin{abstract}
Monotonicity of the likelihood ratio for conditioned densities is a common technical assumption in economic models. But we have found no empirical tests for its plausibility. This paper develops such a test based on the theory of order-restricted inference, which is robust with respect to the correlation structure of the distributions being compared. We apply the test to study the technology revealed by agricultural production experiments. For the data under scrutiny, the results support the assumption of the monotone likelihood ratio. In a second application, we find some support for the assumption of affiliation among bids cast in a multiple-round Vickrey auction for a consumption good.
\end{abstract}

Keywords: affiliation, auction, likelihood ratio, order-restricted inference, stochastic order.

JEL codes: C1, D8, Q0. 


\section{TESTING FOR THE MONOTONE LIKELIHOOD RATIO ASSUMPTION}

The monotone likelihood ratio (MLR) order has arisen in the analysis of many economic problems. When comparing two conditional distributions, the order asserts that a comparatively high outcome is always more likely under one distribution among the pair. The order implies simple stochastic order and is both necessary and sufficient for simple stochastic order on domain-conditioned distribution functions. ${ }^{1}$ This strong level of regularity maps into strongly regular comparative statics inferences; therefore, economic theorists have shown great interest in the assumption.

The list of applications is large, and we will identify a small number to provide a sense of the utility of the assumption. Landsberger and Meilijson (1990) have invoked the assumption to establish insights on the comparative statics of portfolio allocation. In a parallel result, Milgrom (1981a) has established that the MLR order can characterize "bullish" news for an exchange-traded company. Of more direct relevance to the empirical contexts to be studied in this paper, Milgrom (1981a), in extending work by Holmström (1979), has demonstrated the pertinence of the order to mechanism design where parties have different information sets. The types of design issues where it arises include the agent remunerations problem faced by a principal as well as the design of an auction. The assumption is now standard in the theories of organizing (e.g., Koh 1992) and financing (e.g., Innes 1993) firm production under incomplete information.

The intuition behind the assumption is that it makes a signal on an unobservable variable sufficiently informative to be used in a determinate manner when taking signalbased actions. In the instances of principal-agent models, the principal typically does not have sufficient information to remunerate the agent in a first-best manner. For example, while it may be Pareto superior to condition the rewards of a risk-averse agent on effort rather than on production, the principal may not be able to observe the former. If effort shifts the distribution of production according to the MLR order, then we can be confident that a higher level of observed production is evidence of a higher level of agent 
effort. If the principal writes a contract with a reward that is monotone increasing in production, then the agent is likely to respond with more effort, and both parties are better off.

Given that the MLR assumption is often required in contexts where a relevant variable can only be observed after incurring monitoring costs, it is not at all surprising that the assumption has not been subjected to empirical scrutiny. If, however, the remunerated party in a principal-agent relation had no private motive for misrepresenting the relevant variable, then data would be available for study. So, it is typically in economic environments where the MLR assumption is not necessary that the data are available.

There remains, however, the opportunity to test for conjectures on the stochastic relationships underlying data in situations where the private incentive to misrepresent is absent. If we had such data, then a direct test for the validity of the MLR assumption would be possible. In this paper, we develop a direct test of the MLR property and apply it to data from two very different contexts: one from agronomy trials and the other from experimental auctions.

In agronomy trials by academic researchers, the private incentive to misrepresent the level of inputs used is typically absent. Agronomic inputs such as water, fertilizers, and chemical pesticides are tangible, while management time, care in planting, and crop scouting activities are less readily measured. The more measurable among these inputs may be reliably observed by a party with no pecuniary interest in the recorded value who is willing to incur the monitoring costs. In this paper, we use data from carefully controlled agronomy trials at a U.S. land grant university to study the relationship between the nitrogen input and corn (Zea mays) output.

Milgrom and Weber (1982) extended work by Milgrom (1981b) on the structural role of the MLR order in auctions. They characterized equilibrium properties in common and private value auctions when values co-vary across bidders according to the affiliation property. Under this property, which is also known as multivariate totally positive order $2\left(\mathrm{MTP}_{2}\right)$, large values for some of the variables make the other variables more likely (in the likelihood ratio sense) to be large rather than small. In the context of common value auctions and under the assumption of symmetry 
among bidders, a test for the affiliation property coincides with a test for the multivariate extension of the MLR property across conditioned densities. ${ }^{2}$ In our second application, we test for evidence of affiliation in a multivariate valuation distribution where the data has been obtained from second-price auctions. Because of the Vickrey revelation principle for second-price auctions, bidders have no private incentive to bid other than their values. This makes auction data ideal for our test.

After reviewing the literature on testing for stochastic order, we develop our test for the MLR property. As we cannot preclude the possibility that external factors generate correlations between distribution evaluations, the test allows for such dependence. The two empirical applications are then presented. We find strong support for the assumption of MLR order on corn yield distributions as nitrogen application changes. When studying auction experiments, we find limited evidence in favor of the joint hypothesis that agents are symmetric and valuations are affiliated.

\section{Literature Review}

In recent years, several careful tests on stochastic order have been developed, mostly to compare income distributions. Typically, these tests can be adapted to study a variety of stochastic orders. Beach and Davidson (1983) proposed a test of Lorenz curve dominance that explicitly acknowledges that the distribution quantiles are dependent random estimates of the underlying distribution. ${ }^{3}$ However, they treat the compared distributions as independent. Beach and Richmond (1985) applied this method to make Lorenz curve comparisons and to calculate a set of joint confidence intervals about income shares. They adopted a multiple comparison framework in which the distributional properties follow the studentized maximum modulus distribution. Relying on Pearson goodness-of-fit tests, Anderson (1996) used a different approach to infer stochastic dominance. Assuming independence, he derived the asymptotic covariance matrix of the frequency vector for the distributions being compared.

Most of the earlier tests (Beach and Davidson 1983; Anderson 1996) are based on a multiple comparison approach. Partitioning the range of the random variables into a finite set of $k$ exhaustive intervals, the multiple comparison approach assesses the order of the distributions on each of the intervals. These multiple hypotheses tests lead to a statistical 
size bounded by Sidák's inequality, a refinement of Bonferroni’s inequality (Savin 1984, pp. 834-35). This inequality states that a multivariate normal vector with mean zero and arbitrary correlations falls inside a $k$-dimensional cube centered at the origin. In the special case where the correlations are zero, the test statistic has the studentized maximum modulus distribution, a result that Richmond (1982) applied in the construction of simultaneous confidence intervals. Given that these tests are based on Sidák's inequality, and because the correlations may not be zero, the true size of such a multiple comparison test is likely smaller than its nominal size. Kaur, Prakasa Rao, and Singh (1994) have proposed an alternative test for second-order stochastic dominance. ${ }^{4}$ The test, which is valid when observations of each sample are identically independently distributed variables, applies the intersection-union principle.

These types of tests have been criticized because the assumption that compared distributions are independently distributed is often inappropriate for the context in question. This criticism is pertinent for income distribution comparisons that rely on panel data as well as for financial data where different distributions are often conditioned on similar information sets held by the economic agents. With this concern in mind, Klecan, McFadden, and McFadden (1991) introduced a test of stochastic dominance that is an extended version of the Kolmogov-Smirnov test. Their test does not impose a parametric form on the distributions. Through the assumptions of exchangeability and $\alpha$-mixing, the test does allow for some degree of statistical dependence across space and time. Klecan, McFaden, and McFadden derive an upper bound on the size of the test, but the test is likely inefficient because it does not explicitly take into account the dependence structure between distributions.

Davidson and Duclos (2000) demonstrated a theorem that constructs estimates of the asymptotic covariance structure over successive integrals of the probability distributions, that is, the cumulative distribution function (cdf), its integral, and so on. They extended their finding to the case where the quantile of interest, for example, the poverty line, is random. Zheng and Cushing (2001) derived a similar, albeit more restrictive, result and have used it in tests of income inequality indices. Looking again at Lorenz curves, Zheng (2002) developed a procedure for non-simple random samples resulting from stratified and cluster sampling plans. He showed that treating non-simple random samples as 
simple random samples may lead to significant bias in variance-covariance estimates of Lorenz curve coordinates.

As for identifying MLR order, Hendricks, Porter, and Wilson (1990) recognized the need to support their theoretical results on auctions by studying gas lease bids by informed and uninformed bidders. They used a Wilcoxon test to compare the distribution on each percentile, a procedure that does not acknowledge the dependence of distribution quantiles.

Dykstra, Kochar, and Robertson (1995) were the first to develop a formal test for the MLR ordering. They adopted a non-parametric maximum likelihood estimator under the null hypothesis of equality in the probability distribution functions, and their estimator assumes independent random samples. Seeking a general inference platform, Dardanoni and Forcina (1998) proposed a uniform framework for likelihood inference on stochastic orders, for example, the simple stochastic order, the Lorenz order, and the MLR order. Their test leads, after suitable reparameterization, to a system of linear inequalities. They developed a maximum likelihood algorithm based on the expected information matrix, but this estimator relies, again, on the independence of the distributions. Using this estimator of the test statistic, they applied testing procedures for order-restricted inference that rely on results from Robertson, Wright, and Dykstra 1988. In a further extension, Dardanoni and Forcina (1999) adapted their earlier results to compare multiple (that is, more than two) distributions for the case of Lorenz curve orderings. The work of most direct relevance to our auction application is by Bartolucci and Forcina (2000), who developed a likelihood ratio statistic to test for affiliation among binary variables.

In this paper, we develop and apply a test for the MLR order. Our methods generalize to the comparison of three or more distributions, and we develop the test directly in this more general context. Using the parameterization of Dardanoni and Forcina (1998), we derive a test statistic that may be written as a linear combination of inequalities. The asymptotic distribution of the test statistic is derived and, in contrast with Dardanoni and Forcina (1998), we rely on the results of the asymptotic covariance matrix of cdf's as derived in Davidson and Duclos 2000.

Davidson and Duclos extend to the dependent samples context the application by Chow (1989) of the central limit theorem to stochastic inference on dominance orderings 
with independent samples. Their extension is more general than the result applied by Anderson (1996), who uses the asymptotic normality of cell frequency counts associated with two independent samples. Davidson and Duclos confine their attention to the class of stochastic orderings that are generated by comparing successive integrations of cdf's. While the MLR property cannot be presented as such, one of the main theorems in Davidson and Duclos (Theorem 1) can be readily modified through a transformation to apply to the MLR ordering. Our approach is to make this transformation. The asymptotic distribution of our test statistic therefore does not require the assumption of independence among distributions. Dependence is likely the case for economic applications to contract and auction analysis because the distributions to be studied will be conditioned on common environments or common information sets. The derived test statistic, together with the covariance structure, allows us to apply directly the theory of order-restricted regressions to test for the MLR order.

\section{A Nonparametric Test for Stochastic Order}

Let $y \sim F$ be a random variable whose distribution function $y \sim F(y \mid x)$ is conditioned by the decision variable $x$, and whose range is limited to the arbitrarily large closed interval $A \subset \mathbb{R}$. To set up notation for the statistical test, we divide the range into a partition characterized by the completely ordered vector $\left(a_{1}, a_{2}, \ldots, a_{k}\right)$ and we let $a_{0}=$ $\inf (\mathrm{A})$. The probability vector $p_{i, j}=\operatorname{Pr}\left(y \in\left[a_{j-1}, a_{j}\right] \mid x=x_{i}\right)$ denotes the conditional probability that $y$ falls into the $j$ th interval given that the decision variable takes the value $x_{i}$. Let $p_{i}=\left(p_{i, 1}, p_{i, 2}, \ldots, p_{i, k}\right)^{\prime}$ with $p_{i, k+1}=1-\sum_{j=1}^{k} p_{i, j}$ so that $p_{i}$ fully describes the discrete approximation of the conditional distribution. This notation allows us to describe the MLR order in the following manner.

DEFINITION 1. Random variable $y_{i+1} \sim F\left(y \mid x_{i+1}\right)$ is said to dominate random variable $y_{i} \sim$ $F\left(y \mid x_{i}\right)$ according to the monotone likelihood ratio order if, for any partition of the range, $p_{i, j} / p_{i, j+1} \geq p_{i+1, j} / p_{i+1, j+1} \forall j \in\{1,2, \ldots, k\}$. In this case, we write $y_{i+1} \succeq_{r} y_{i}$. 
The definition carries over to the case where more than two distributions are compared. If we let $i=\{1,2, \ldots, m+1\}$ index the distributions of interest, then we say that a sequence of distributions is stochastically ordered in the MLR sense whenever

$$
y_{m+1} \succeq_{r} y_{m} \succeq_{r} \ldots \succeq_{r} y_{1} \text {. }
$$

When testing for stochastic order among distribution functions, randomness arises because the sample data only imperfectly represent the underlying true population distribution. A testing procedure should take this randomness into account. It should also acknowledge the possibility of dependence through common conditioning environmental and economic factors. Many of the tests for stochastic orderings available in the literature are ill suited to model the typical economic environment because these tests suppose that samples are independently drawn. We overcome this problem by adapting a theorem in Davidson and Duclos 2000 to develop a test that takes explicit account of the covariance structure between conditional distributions. This allows us to derive the asymptotic distributions of the variables of interest. We can then apply results for order-restricted inference (see Robertson, Wright, and Dykstra 1988) to test for the MLR order.

This section is structured as follows. After preparing the test statistic, we derive its asymptotic distribution. Then we introduce the concepts of order-restricted inference that allow us to proceed with a one-sided test for the MLR order.

\section{A Presentation of Data for Hypothesis Testing}

The hypothesis to be tested involves a comparison of $m+1$ conditional empirical distribution functions, and the data processing algorithm to be used treats the data as a single set. For the $\mathbf{p}_{\mathbf{i}}$ associated with the $m+1$ evaluations of the conditioning variable, form a $k \times(m+1)$ matrix, and denote it by $\mathbf{p}$. Identify by $\operatorname{vec}(\mathbf{p})$ the $(m+1) k \times 1$ vector formed by stacking the columns in $\mathbf{p}$. Denote the $k \times k$ identity matrix by $I_{k}$, and denote the upper triangular matrix of 1's by $T_{k}$. Observe that the inverse of the latter, $T_{k}^{-1}$, has 1 's on the diagonal and -1 's on the first upper diagonal. Also, define $D_{m}$ as the $m \times(m+1)$ matrix with entries $d_{i, j}$ where, $d_{i, i}=1, d_{i, i+1}=-1$, and $d_{i, j}=0$ otherwise.

Define $\theta_{i, j}=\ln \left(p_{i, j} / p_{i, k+1}\right)$ so that $\theta_{i, j}$ is the multivariate logistic transformation of $\mathbf{p}_{\mathbf{i}}$. Collect these $\theta_{i, j}$ evaluations into a $k \times(m+1)$ matrix, and call it $\Theta$. Note that 
$\ln \left(p_{i, j} / p_{i, j+1}\right)=\theta_{i, j}-\theta_{i, j+1}$ for $j<k$, and $\ln \left(p_{i, j} / p_{i, j+1}\right)=\theta_{i, k}$ for $j=k$. Following

Dardanoni and Forcina (1998), the MLR order implies

$$
\mathbf{b}_{\mathbf{r}}=\left(D_{m} \otimes T_{k}^{-1}\right) \operatorname{vec}(\Theta) \geq 0
$$

where premultiplication by matrix $T_{k}^{-1}$ replaces the numéraire residual probability with an adjacent probability in the ratios given by $\theta_{i, j}$, and where $D_{m}$ generates comparisons across the distributions under consideration.

\section{Asymptotic Properties of the Test Statistic}

Davidson and Duclos (2000) present a theorem, adapted to our case as Lemma 1 in Appendix A, that identifies the asymptotic properties of two correlated cdf's for two samples of size $n$. Using their result on the asymptotic properties of the cdf estimates and using the delta method (Lemma 2, Appendix A), we can derive the asymptotic properties of the statistics $\mathbf{b}_{\mathbf{r}}$. Let vector $\mathbf{F}_{\mathbf{i}}$ have the $j$ th ordinate $F_{i, j}=\operatorname{Pr}\left(y \leq a_{j} \mid x_{i}\right)=\sum_{l=1}^{j} p_{i, 1}$, $j=1,2, \ldots, k$. For the $\mathbf{F}_{\mathbf{i}}$ associated with the $m+1$ conditioning variables, form a $k \times(m+$ 1) matrix, and denote it by $\mathbf{F}$. Let $\operatorname{vec}(\mathbf{F})$ be the $(m+1) k \times 1$ vector that is obtained after stacking the columns in sequence where $\mathbf{F}_{\mathbf{1}}$ provides the first $k$ entries.

Proposition 1. Let $\Sigma$ be the covariance matrix of vec $(\mathbf{F})$. Define, for $i \in\{1,2, \ldots, m\}$, the $k \times k$ matrix

$$
C_{r, i}=\left(\begin{array}{ccccc}
\frac{p_{i, 1}+p_{i, 2}}{p_{i, 1} p_{i, 2}} & -\frac{1}{p_{i, 2}} & 0 & \cdots & 0 \\
-\frac{1}{p_{i, 2}} & \frac{p_{i, 2}+p_{i, 3}}{p_{i, 2} p_{i, 3}} & -\frac{1}{p_{i, 3}} & \cdots & 0 \\
0 & -\frac{1}{p_{i, 3}} & \frac{p_{i, 3}+p_{i, 4}}{p_{i, 3} p_{i, 4}} & \cdots & 0 \\
\vdots & \vdots & \vdots & \ddots & \vdots \\
0 & 0 & 0 & \cdots & \frac{p_{i, k}+p_{i, k+1}}{p_{i, k} p_{i, k+1}}
\end{array}\right),
$$

and define the $m k \times(m+1) k$ matrix 


$$
C_{r}=\left(\begin{array}{ccccc}
C_{r, 1} & -C_{r, 2} & 0 & \ldots & 0 \\
0 & C_{r, 2} & -C_{r, 3} & \ldots & 0 \\
\vdots & \vdots & \vdots & \ddots & \vdots \\
0 & 0 & 0 & \ldots & -C_{r, m+1}
\end{array}\right)
$$

Then the $m k \times 1$ vector $\sqrt{n}\left(\hat{\mathbf{b}}_{\mathbf{r}}-\mathbf{b}_{\mathbf{r}}\right)$ has asymptotic normal distribution with mean zero and covariance matrix $\Sigma_{r}=C_{r} \Sigma C_{r}^{\prime}$.

The proof is provided in Appendix B. Next, we pose the hypothesis that we seek to test.

\section{Hypothesis Testing}

We wish to test for $H_{0}$ over $H_{1}$ where

$H_{0}: \mathbf{b}_{\mathbf{r}} \geq 0$ (weak order);

$H_{1}$ : (the distributions are not ordered in the sense of $H_{0}$ ).

The test is based on a distance statistic and has been developed in the context of order-restricted inference by Robertson, Wright, and Dykstra (1988), among others. Let $\Gamma$ denote a convex cone contained in $\mathbb{R}^{v}$, and let the positive definite $v \times v$ matrix $\mathbf{V}$ describe a metric. Then the distance between a vector $\mathbf{z} \in \mathbb{R}^{v}$ and set $\Gamma$ is defined as

$$
d(\mathbf{z}, \Gamma, \mathrm{V})=\inf _{\tilde{\mathbf{z}} \in \Gamma}(\mathbf{z}-\tilde{\mathbf{z}})^{\prime} \mathrm{V}^{-1}(\mathbf{z}-\tilde{\mathbf{z}})
$$

Viewing the null and alternative hypotheses as subspaces, $H_{0}, H_{1} \subset \mathbb{R}^{v}$, the distance statistic may be written as

$$
D_{0,1}=n\left\{d\left(\hat{\mathbf{b}}_{\mathbf{r}}, H_{0}, \mathrm{~V}\right)-d\left(\hat{\mathbf{b}}_{\mathbf{r}}, H_{0} \cup H_{1}, \mathrm{~V}\right)\right\}
$$

The distance statistic equals zero whenever the sample value of $\hat{\mathbf{b}}_{\mathbf{r}}$ is consistent with the null hypothesis. For $\sqrt{n}\left(\hat{\mathbf{b}}_{\mathbf{r}}-\mathbf{b}_{\mathbf{r}}\right) \stackrel{a}{\sim} N\left(0, \Sigma_{r}\right)$, the test statistic may be described as

$$
H_{0} \text { against } H_{1}: D_{0,1}=\min _{\hat{\mathbf{b}}_{\mathbf{r}} \geq 0} n\left(\hat{\mathbf{b}}_{\mathbf{r}}-\tilde{\mathbf{b}}_{\mathbf{r}}\right)^{\prime} \Sigma_{r}^{-1}\left(\hat{\mathbf{b}}_{\mathbf{r}}-\tilde{\mathbf{b}}_{\mathbf{r}}\right)
$$

Problem (6) is solved through quadratic programming and the covariance matrix is estimated by its sample equivalent. Test statistic $D_{0,1}$ is $\bar{\chi}^{2}$ distributed such that 
$\operatorname{Pr}\left(D_{0,1} \geq c\right)=\sum_{\mathrm{l}=0}^{m k} \operatorname{Pr}\left(\chi_{l}^{2} \geq c\right) w\left(m k, m k-l, \Sigma_{r}\right)$, and the null hypothesis is evaluated at its least-favorable alternative, that is, at $\mathbf{b}_{\mathbf{r}}=0$ (Wolak 1989, p. 214). The $\bar{\chi}^{2}$ distribution is a mixture of weighted $\chi$ distributions where the $\chi_{0}^{2}$ distribution is defined as the point mass at the origin. The weights given by $w\left(m k, m k-l, \Sigma_{r}\right)$ are the probabilities that vector $\mathbf{b}_{\mathbf{r}}$ has exactly $m k-1$ positive elements where, by definition, these probabilities sum to 1 . The exact critical values depend explicitly on covariance matrix $\Sigma_{r}$ and are difficult to determine for $k>0$ (see Kudô 1963 for the closed-form solution formulae when $k \leq 4$ ).

Following Wolak (1989), we determine these values through Monte Carlo simulations. Drawing 1,000 times from the multivariate normal distribution with mean 0 and covariance matrix $\Sigma_{r}$, vector $\tilde{\mathbf{b}}_{\mathbf{r}}$ is computed for each draw. The weights are the proportions of the 1,000 draws such that $\tilde{\mathbf{b}}_{\mathbf{r}}$ has exactly $l$ positive elements. All covariance matrices are estimated by their sample equivalents.

\section{Application to Production Technology}

Site-level information on the use of nitrogen and other inputs is not available to crop insurance companies. While these inputs are tangible and purchases of artificial sources of nitrogen can be documented, the inputs can be stored for future use, sold or bartered on secondary markets, or applied on land other than that planted under the insured crop. ${ }^{5}$ Thus, crop insurers are posed with a moral hazard problem that can be avoided only by incurring prohibitively large monitoring costs. Chambers (1989) has shown that the MLR order plays an important role in understanding the moral hazard constraints that a crop insurer faces when designing a contract. Specifically, with an action-induced MLR dominance order and constant absolute risk aversion, the risk-neutral insurer will leave the grower with a positive fraction of incremental crop revenue. In this way, incomplete risk shifting is used to encourage input use by the grower. ${ }^{6}$ The moral hazard problem would appear to be particularly severe in crop agriculture. Babcock and Hennessy (1996), among others, have identified moral hazard as one of the main reasons for failure of commercial crop insurance markets in the United States and elsewhere. ${ }^{7}$ 


\section{Data}

Data were obtained from Iowa corn plots grown by the nitrogen research team in the Department of Agronomy at Iowa State University during the five-year period 1987-91. The data previously has been described in Babcock and Hennessy (1996). Four geographically dispersed farms sites were involved, and ten different nitrogen application levels, ranging from 0 to $300 \mathrm{lb} / \mathrm{acre}$, were applied. The rates were given in $25 \mathrm{lb} / \mathrm{acre}$ increments at low application rates and in $50 \mathrm{lb} /$ acre increments at higher application rates. The data set consists of 600 observations where there are 60 observations per nitrogen application rate. In order to aggregate across years and sites, we correct the distributions by year and site factors. The details are provided in Appendix C.

The input-conditioned empirical distributions of corn yields that we compare are the nine pairs of adjacent nitrogen applications rates: $0 \mathrm{lb} / \mathrm{acre}$ versus $25 \mathrm{lb} / \mathrm{acre}$ through to $250 \mathrm{lb} /$ acre versus $300 \mathrm{lb} / \mathrm{acre}$. To form the partition of the yield range, the vectors of the distributions (two or more) to be compared are stacked and then divided into $k+1=5$ equiprobable intervals. Then, using these intervals, the empirical cdf is calculated at each nitrogen application rate. The number of intervals was chosen in order to ensure that each cell receives a strictly positive probability weight for each of the distributions being compared.

\section{Results}

Note that a rejection of $H_{0}$, implying that $y_{i+1}$ does not MLR dominate $y_{i}$, does not imply that $y_{i}$ MLR dominates $y_{i}+1$. But the comparison of distributions certainly can be turned around to test for the latter relationship. We do so, and consequently we report two sets of results. Because the random variables can be exchanged in this manner, the $\tilde{\mathbf{b}}_{\mathbf{r}}$ vector for the test in one direction is the additive inverse of the test in the reverse direction.

Table 1 presents the results when comparing two distributions. The first set of columns (columns 1 and 2) shows pairs of nitrogen application rates, $i$ and $i+1$, for which the distributions are compared. The second set of columns (columns 3 and 4) is labeled $y_{i+1} \succeq_{r} y_{i}$, and compares the distributions in the sense that the yield distribution of the higher nitrogen application level dominates the yield distribution of the lower 
TABLE 1. Order-restricted test for the likelihood ratio order

\begin{tabular}{|c|c|c|c|c|c|}
\hline \multicolumn{2}{|c|}{$\begin{array}{c}\text { Nitrogen } \\
\text { Rate } \\
\text { (lb/acre) }\end{array}$} & \multicolumn{2}{|c|}{ Hypothesis $H_{0}: y_{i+1} \succeq_{r} y_{i}$} & \multicolumn{2}{|c|}{ Hypothesis $H_{0}: y_{i} \succeq_{r} y_{i+1}$} \\
\hline$i$ & $i+1$ & $\begin{array}{c}\text { Distance } \\
\text { Statistic, } \\
D_{0,1}\end{array}$ & $\begin{array}{c}\bar{\chi}_{0,1}^{2} \text { Distribution, } \\
\text { Critical Values } \\
\text { at } \alpha=0.1,0.05, \\
0.01\end{array}$ & $\begin{array}{c}\text { Distance } \\
\text { Statistic, } \\
D_{0,1}\end{array}$ & $\begin{array}{c}\bar{\chi}_{0,1}^{2} \text { Distribution, } \\
\text { Critical Values } \\
\text { at } \alpha=0.1,0.05, \\
0.01\end{array}$ \\
\hline 0 & 25 & 0 & $6.31,7.84,11.98$ & $26.79^{* * * *}$ & $6.33,7.87,11.40$ \\
\hline 25 & 50 & 0.11 & $6.39,7.95,11.55$ & $21.05^{* * *}$ & $6.29,7.83,11.37$ \\
\hline 50 & 75 & 1.85 & $6.31,7.85,11.39$ & $33.88^{* * *}$ & $6.23,7.87,11.40$ \\
\hline 75 & 100 & 0 & $6.34,7.89,11.45$ & $8.20^{* *}$ & $6.27,7.81,11.33$ \\
\hline 100 & 125 & 0.13 & $6.29,7.82,11.34$ & $20.38^{* * * *}$ & $6.22,7.74,11.23$ \\
\hline 125 & 150 & 1.45 & $6.26,7.77,11.27$ & 4.78 & $6.26,7.78,11.28$ \\
\hline 150 & 200 & 4.57 & $6.33,7.86,11.27$ & $10.99^{* *}$ & $6.25,7.77,11.27$ \\
\hline 200 & 250 & 0.36 & $6.33,7.86,11.38$ & $9.17^{* *}$ & $6.35,7.89,11.44$ \\
\hline 250 & 300 & $7.37^{*}$ & $6.23,7.74,11.24$ & 2.50 & $6.22,7.73,11.42$ \\
\hline
\end{tabular}

nitrogen application level. The third set of columns (columns 5 and 6 ) is labeled $y_{i} \succeq_{r} y_{i+1}$, and compares the distributions in the reverse direction. Columns 3 and 5 report the test statistic for $H_{0}$ against $H_{1}$. The $0.1,0.05$, and 0.01 critical values of the $\bar{\chi}_{0,1}^{2}$ distribution are reported in columns 4 and 6 , for the respective comparisons. The exact value depends on the covariance matrix $\Sigma_{r}$, and so the values depend on the pair of distributions under comparison.

We observe in column 3 that $H_{0}$ is not rejected in favor of $H_{1}$ for nitrogen applications between $0 \mathrm{lb} /$ acre and $250 \mathrm{lb} / \mathrm{acre}$ at any significance level. This provides strong evidence in favor of the MLR order. These results are again confirmed in the reverse comparison in Table 1. Here the MLR order, when comparing distributions in the direction of decreasing order, is rejected in favor of $H_{1}$ (no order) at the 0.05 significance level in seven of the nine comparisons. The exceptions are for the comparison of $150 \mathrm{lb} / \mathrm{acre}$ versus $125 \mathrm{lb} / \mathrm{acre}$ and for the comparison of $300 \mathrm{lb} / \mathrm{acre}$ versus $250 \mathrm{lb} / \mathrm{acre}$. In Iowa the commercial nitrogen application rate is typically between 100 and $200 \mathrm{lb} / \mathrm{acre}$ (Duffy and Smith 2002), and commercial rates have not varied much over the past 20 years. 
In microeconomic analysis, it is common to assume that the MLR order holds globally. We test for this hypothesis over the economically relevant range of fertilizer applications from 100 to $200 \mathrm{lb} /$ acre where $m=3$. The hypothesis $y_{200} \succeq_{r} y_{150} \succeq_{r} y_{125} \succeq_{r} y_{100}$ generates the test statistic value 4.14 and is not rejected at any conventional significance level. ${ }^{8}$ However, the reverse hypothesis, with a test statistic of 40.77 , is rejected at the 1 percent significance level. ${ }^{9}$

\section{Application to Auctions}

Interest in the use of laboratory auctions for evaluating decision making, for assessing the acceptance of new products, and for understanding reactions to publicly posted information has grown. Many of these auctions proceed in multiple rounds and post the prevailing market price after every trial. This procedure allows newcomers to gain experience in the "market." Concern exists that posting price information could alter bids when valuations are correlated in the affiliation sense. In our second application, we show how the test can be applied to the hypothesis of an affiliated, symmetric distribution of valuations, which is one of the principal assumptions underlying the standard auctions model. Because the affiliation property coincides with the multivariate extension of the MLR ordering when the bidders are symmetric, if posting the price is found to shift bid distributions in the MLR sense, it would provide strong evidence that the underlying values are drawn from an affiliated distribution.

Testing for the property has been of interest in common value auctions under asymmetric information. ${ }^{10}$ In empirical studies of oil and gas lease auctions, Hendricks, Porter, and Wilson (1990) tested for MLR order on the bid distributions among informed and uninformed bidders. The test supported their assumption of an MLR dominating shift between bid data from informed and uninformed bidders.

Possible value affiliation has been subject to a critical assessment in private-value, multiple-round Vickrey auctions with price posting after every round. As agents extract information on value perceptions from price formation in the market, price posting will allow buyers with affiliated values to update their values iteratively as evidence mounts to allay fears of overbidding. List and Shogren (1999) employed least-squares methods to ascertain how intermediate-round price information affects bidding behavior in multiple- 
round auctions. Data were drawn from auction experiments evaluating willingness to pay for pathogen-screened meat sandwiches. They found that posted prices (1) do not affect behavior of the median experience bidder, but (2) do influence the behavior of the median bidder among bidders unfamiliar with a product. In contrast to List and Shogren (1999), who tested for linear correlation between posted price and the median bid in the subsequent round, we apply our methods to test for the MLR order on distributions of bids cast in consecutive rounds. ${ }^{11}$

\section{Data}

We use data from a laboratory auction in which participants were asked for their willingness to pay to exchange a typical meat sandwich with a sandwich that has been carefully screened for either Campylobacter or Salmonella contamination (Shogren et al. 1994). The solicitation mechanism was a second-price, multiple-round auction where each group consisted of 15 bidders. The prevailing market price was posted after each round. The auction consisted of 10 rounds of naive bids, where subjects did not receive any information on the risk involved in Campylobacter/Salmonella infection. However, subjects were informed that the stringently screened sandwich posed a probability of illness that amounts to 1 in 100 million. Some summary statistics are provided in Table 2, where we show the median bid alongside the posted price (the second-highest bid) in each round. The prevailing market price varies between $\$ 1.00$ and $\$ 1.38$ for the sandwich screened for Campylobacter and between $\$ 0.63$ and $\$ 1.50$ for the sandwich screened for Salmonella.

\section{Results}

In our data analysis we chose $k=4$. Because the conditioning variables- the posted prices - do not necessarily change monotonically along the sequence of trials, we compare only pairs of distributions $(m=1)$. Table 2 shows the results in the fourth and fifth columns. Of the 36 comparisons, we find only two rejections, one in the case of Campylobacter and one in the case of Salmonella. This means that a statistically significant MLR ordering on the distribution shift cannot be discerned for most comparisons. 
TABLE 2. Auction bid distribution after price posting

\begin{tabular}{|c|c|c|c|c|c|c|}
\hline Trial & Min & Median & Max & Price & $H_{0}: y_{i+1} \succeq_{r} y_{i}$ & $H_{0}: y_{i} \succeq_{r} y_{i+1}$ \\
\hline \multicolumn{7}{|c|}{ Campylobacter } \\
\hline$i=1$ & 0.05 & 0.50 & 2.00 & 1.00 & 4.72 & 4.34 \\
\hline$i=2$ & 0.05 & 0.50 & 1.80 & 1.00 & 4.73 & $6.60^{*}$ \\
\hline$i=3$ & 0.05 & 0.50 & 1.50 & 1.25 & 3.15 & 3.34 \\
\hline$i=4$ & 0.00 & 0.50 & 2.00 & 1.15 & 3.92 & 4.20 \\
\hline$i=5$ & 0.05 & 0.50 & 1.45 & 1.25 & 1.96 & 3.55 \\
\hline$i=6$ & 0.05 & 0.70 & 1.50 & 1.38 & 2.29 & 2.13 \\
\hline$i=7$ & 0.05 & 0.65 & 1.50 & 1.35 & 2.18 & 2.14 \\
\hline$i=8$ & 0.00 & 0.50 & 1.50 & 1.25 & 2.24 & 2.14 \\
\hline$i=9$ & 0.00 & 0.55 & 1.45 & 1.25 & 3.60 & 3.07 \\
\hline$i=10$ & 0.00 & 0.50 & 1.40 & 1.15 & & \\
\hline \multicolumn{7}{|c|}{ Salmonella } \\
\hline$i=1$ & 0.08 & 0.50 & 2.00 & 1.50 & 6.25 & $6.77^{*}$ \\
\hline$i=2$ & 0.10 & 0.50 & 1.45 & 1.00 & 5.43 & 3.54 \\
\hline$i=3$ & 0.10 & 0.50 & 1.40 & 0.80 & 3.59 & 2.95 \\
\hline$i=4$ & 0.11 & 0.47 & 1.8 & 1.25 & 2.00 & 2.81 \\
\hline$i=5$ & 0.10 & 0.50 & 1.30 & 0.80 & 2.15 & 2.17 \\
\hline$i=6$ & 0.10 & 0.50 & 1.00 & 0.92 & 5.59 & 4.65 \\
\hline$i=7$ & 0.10 & 0.47 & 0.78 & 0.75 & 2.30 & 3.01 \\
\hline$i=8$ & 0.10 & 0.50 & 0.90 & 0.75 & 2.45 & 0.30 \\
\hline$i=9$ & 0.10 & 0.50 & 0.87 & 0.80 & 2.72 & 2.27 \\
\hline$i=10$ & 0.10 & 0.50 & 0.80 & 0.63 & & \\
\hline
\end{tabular}

Note: * denotes significance at the 0.1 level. Parameter $k$ has value 4.

The two instances where we identify the MLR order are the comparison of trials 2 and 3 in the instance of Campylobacter and in the case of trials 1 and 2 in the instance of Salmonella. For both pathogens, the price signal has shifted the distribution upwards in the MLR sense. That is, the price information was taken by the subjects to be a positive signal, "good news" in the words of Milgrom (1981a).

Our results confirm those of List and Shogren (1999). For newcomers evaluating unfamiliar goods, price posting might lead to weak affiliation of the bid distribution. If posted prices were interpreted as informative signals on value, we would expect this to be 
the case early in the auction. However, the affiliation effect disappears after trial 3 when the information-adjusted values settle to a new equilibrium.

\section{Conclusion}

In this paper, we have presented a robust procedure for testing for stochastic order among distributions that takes explicit account of the correlation structure of the distributions being compared. Using agricultural crop trials data, we applied the test to identify stochastic order in the likelihood ratio sense. Our results support the hypothesis of MLR order over a large range of input levels. We conclude that the MLR assumption commonly made in theoretical work is confirmed for our data set. In a second application, we studied private evaluations of a new good in a multiple-round Vickrey auction to identify some evidence in favor of the affiliated values assumption commonly employed in the economics of imperfect information.

Other stochastic orders also arise in economic models. The test can be extended to accommodate alternative univariate stochastic orders such as the reverse hazard (monotone probability ratio) order, which has arisen in portfolio theory (Athey 2002) and elsewhere. 


\section{Endnotes}

1. Under the simple stochastic order, the compared distributions cannot intersect and the dominating distribution lies underneath the dominated distribution. Under the MLR order, the ratio of the densities, say $f_{2}(x) / f_{1}(x)$ where $f_{2}(x)$ dominates, is monotone increasing where defined. Graphically, one implication of the MLR ordering is that the ordered densities can cross no more than once.

2. The affiliation property is defined on lattice-structured sets. We direct the interested reader to Athey 2002. See Shaked and Shanthikumar 1994 (p. 132 and p. 253) on the relation between the multivariate MLR order and the affiliation property. It suffices to test for the MLR property instead of for affiliation because the standard model in auction theory assumes symmetry across agent strategies and beliefs so that all agents have the same probability function on valuations. Bartolucci and Forcina (2000), in contrast, have developed a likelihood ratio test for the $\mathrm{MTP}_{2}$ property on a distribution of binary variables. Such a test, were it extended to continuous variables, would make it possible to dispense with the symmetry condition in deriving the test hypothesis.

3. As Lorenz curves are constructed with share data, Lorenz-curve dominance may be interpreted as a mean-preserving contraction in the variability of income shares.

4. An increase in this order increases the expected value of all increasing and concave functions.

5. Farmers typically rotate land through different crops in order to disrupt the progression of pest infestations, to replenish soil nutrients, and to rest ground from intensive cultivation practices. For corn in the midwestern United States, the most common rotations are corn-soybean and corn-corn-soybean.

6. Were risk-neutral insurers perfectly informed, then they would have assumed all the risk. Thus, the equilibrium contract is not optimal but it is an informationconstrained optimum.

7. Commercial crop insurance markets do exist in the United States, but their viability is underpinned by government subsidies. Also, the U.S. federal government has at times made crop insurance coverage a pre-requisite in determining eligibility for agricultural income support.

8. The critical values are $11.95,14.01$, and 18.41 at the $0.1,0.05$, and 0.01 significance levels. 
9. The critical values are $11.98,14.05$, and 18.45 at the respective significance levels $0.1,0.05$, and 0.01 .

10. A common value auction is one in which all bidders place equal, but unknown, value on an asset. A private value auction is one in which bidders place different values on the asset.

11. As the auctions are Vickrey, the bids reveal the valuations. Therefore, we are testing for the MLR ordering on conditioned distributions of valuations. 


\section{Appendix A: Lemmas 1 and 2}

Lemma 1 is adapted from Davidson and Duclos 2000 (p. 1444) when applied to cdf's. Let $\hat{F}_{i}(y)$ be the empirically observed cumulative distribution corresponding to the true distribution $F_{i}(y)$. Define function $\operatorname{Ind}\left(y^{i} \leq y\right)$ as the indicator function, assuming value 1 when the argument is true and 0 otherwise, and let expression $E\left[\operatorname{Ind}\left(y^{A} \leq y\right) \operatorname{Ind}\left(y^{B} \leq y^{\prime}\right)\right]$ be the unconditional expectation on the product of indicator functions for random variables $y^{A}$ and $y^{B}$.

LEMMA 1. For $i \in\{A, B\}$, the statistic $\sqrt{n}\left[\hat{F}_{i}(y)-F_{i}(y)\right]$ is asymptotically normal with mean zero and asymptotic covariance structure given by

$$
\lim _{n \rightarrow \infty} n \operatorname{Cov}\left[\hat{F}_{A}(y), \hat{F}_{B}\left(y^{\prime}\right)\right]=E\left[\operatorname{Ind}\left(y^{A} \leq y\right) \operatorname{Ind}\left(y^{B} \leq y\right)\right]-F_{A}(y) F_{B}\left(y^{\prime}\right) .
$$

The covariance matrix $\Sigma=\left(\sigma_{i, j}\right)$ may be consistently estimated from the empirical distribution function (Davidson and Duclos 2000, p. 1445)

$$
\hat{\sigma}_{i, j}=n^{-1} \sum_{m=1}^{n} \operatorname{Ind}\left(y_{m}^{A} \leq y\right) \operatorname{Ind}\left(y_{m}^{B} \leq y^{\prime}\right)-\hat{F}_{i}(y) \hat{F}_{j}\left(y^{\prime}\right),\{i, j\} \in\{A, B\} .
$$

Lemma 2, below, provides the delta method (Greene 2000, p. 118), which we use to establish asymptotic distributions.

LEMMA 2. If $z_{n}$ is a $K \times 1$ sequence of vector-valued random variables such that

$\sqrt{n}\left(z_{n}-\mu\right) \stackrel{d}{\rightarrow} N(0, \Sigma)$, and if $c\left(z_{n}\right)$ is a set of $J$ continuous functions of $z_{n}$, not involving $n$, then 


$$
\sqrt{n}\left[c\left(z_{n}\right)-c(\mu)\right] \stackrel{d}{\rightarrow} N\left[0, C(\mu) \Sigma C(\mu)^{\prime}\right]
$$

where $C(\mu)$ is the $J \times K$ matrix given by $\partial c(\mu) / \partial \mu^{\prime}$. 


\section{Appendix B: Proof of Proposition 1}

The test statistic is given by $\mathbf{b}_{\mathbf{r}}=\left(D_{m} \otimes T_{k}^{-1}\right) \operatorname{vec}(\Theta)$ where $\theta_{i, j} \ln \left(p_{i, j} / p_{i, k+1}\right)=$ $\ln \left[\left(F_{i, j}-F_{i, j-1}\right) /\left(1-F_{i, k}\right)\right]$. Hence $\mathbf{b}_{\mathbf{r}}=$

$$
\begin{aligned}
& \begin{array}{c}
\left(\begin{array}{c}
\ln \left(\frac{F_{1,1}}{1-F_{1, k}}\right)-\ln \left(\frac{F_{1,2}-F_{1,1}}{1-F_{1, k}}\right)-\ln \left(\frac{F_{2,1}}{1-F_{2, k}}\right)+\ln \left(\frac{F_{2,2}-F_{2,1}}{1-F_{2, k}}\right) \\
\ln \left(\frac{F_{1,2}-F_{1,1}}{1-F_{1, k}}\right)-\ln \left(\frac{F_{1,3}-F_{1,2}}{1-F_{1, k}}\right)-\ln \left(\frac{F_{2,2}-F_{2,1}}{1-F_{2, k}}\right)+\ln \left(\frac{F_{2,3}-F_{2,2}}{1-F_{2, k}}\right) \\
\vdots \\
\ln \left(\frac{F_{1, k}-F_{1, k-1}}{1-F_{1, k}}\right)-\ln \left(\frac{F_{2, k}-F_{2, k-1}}{1-F_{2, k}}\right) \\
\vdots \\
\ln \left(\frac{F_{m, 1}}{1-F_{m, k}}\right)-\ln \left(\frac{F_{m, 2}-F_{m, 1}}{1-F_{m, k}}\right)-\ln \left(\frac{F_{m+1,1}}{1-F_{m+1, k}}\right)+\ln \left(\frac{F_{m+1,2}-F_{m+1,1}}{1-F_{m+1, k}}\right) \\
\ln \left(\frac{F_{m, 2}-F_{m, 1}}{1-F_{m, k}}\right)-\ln \left(\frac{F_{m, 3}-F_{m, 2}}{1-F_{m, k}}\right)-\ln \left(\frac{F_{m+1,2}-F_{m+1,1}}{1-F_{m+1, k}}\right)+\ln \left(\frac{F_{m+1,3}-F_{m+1,2}}{1-F_{m+1, k}}\right) \\
\vdots \\
\ln \left(\frac{F_{m, k}-F_{m, k-1}}{1-F_{m, k}}\right)-\ln \left(\frac{F_{m+1, k}-F_{m+1, k-1}}{1-F_{m+1, k}}\right)
\end{array}\right)
\end{array} \\
& =\left(\begin{array}{c}
\ln \left(F_{1,1}\right)-\ln \left(F_{1,2}-F_{1,1}\right)-\ln \left(F_{2,1}\right)+\ln \left(F_{2,2}-F_{2,1}\right) \\
\ln \left(F_{2,2}-F_{2,1}\right)+\ln \left(F_{2,3}-F_{2,2}\right) \\
\ln \left(F_{1,2}-F_{1,1}\right)-\ln \left(F_{1,3}-F_{1,2}\right)-\ln \left(F_{2, k}\right) \\
\vdots \\
\ln \left(F_{1, k}-F_{1, k-1}\right)-\ln \left(1-F_{1, k}\right)-\ln \left(F_{2, k}-F_{2, k-1}\right)+\ln \left(1-F_{2, k}\right) \\
\operatorname{co} \\
\ln \left(F_{m, 1}\right)-\ln \left(F_{m, 2}-F_{m, 1}\right)-\ln \left(F_{m+1,1}\right)+\ln \left(F_{m+1,2}-F_{m+1,1}\right) \\
\operatorname{c}\left(F_{m+1,2}-F_{m+1,1}\right)+\ln \left(F_{m+1,3}-F_{m+1,2}\right) \\
\ln \left(F_{m, 2}-F_{m, 1}\right)-\ln \left(F_{m, 3}-F_{m, 2}\right)-\ln \left(F_{m+1, k}\right)+\ln \left(1-F_{m+1, k}\right)
\end{array}\right) .
\end{aligned}
$$


Because the covariance structure of the cdf's can be estimated using Lemma 1, (B.1) will allow asymptotic estimation of the test-statistic moments. Noting that $F_{i, 1}=p_{i, 1}, i \in\{1,2, \ldots, m+1\}$ and $F_{i, j}-F_{i, j-1}=p_{i, j}, i \in\{1,2, \ldots, m+1\}, j \in\{2,3, \ldots, k\}$, the derivative of $\mathbf{b}_{\mathbf{r}}$ with respect to $\mathbf{F}, \partial \mathbf{b}_{\mathbf{r}} / \partial \mathbf{F}$, may be written as the $m k \times(m+1) k$ matrix given in equation (3). The result follows upon applying Lemma 2. 


\section{Appendix C: Method for Correcting Distributions in the Data Set}

A statistical analysis of Iowa corn yields at the state level identifies a clear time trend in the data because of technical progress over the period 1973-99. To control for this, we postulate a deterministic component of yield depending on time, as well as on-site effects and a random component. To account for changes in yield because of technical progress, the data is corrected by estimating a linear time trend for Iowa corn yields from Iowa average yield over the period 1973-99 (Iowa Dept. of Agriculture various). Corn yields are estimated to increase by $1.753 \mathrm{bu} / \mathrm{acre} / \mathrm{year}$ where the regression $\mathrm{R}^{2}$ is 0.389 and the parameter to the linear time trend has a $t$-value of 3.986. Using the trend-corrected yield data, we tested for equality in means across sites. We rejected the hypothesis of equality at the 1 percent level, $F>F_{1,595}=8.61$. For this reason, we proceeded by correcting for the mean differences across sites. 


\section{References}

Anderson, G. 1996. "Nonparametric Tests of Stochastic Dominance in Income Distributions." Econometrica 64(September): 1183-93.

Athey, S. 2002. "Monotone Comparative Statics Under Uncertainty." Quarterly Journal of Economics 117(February): 187-223.

Babcock, B. A., and D. A. Hennessy. 1996. "Input Demand under Yield and Revenue Insurance." American Journal of Agricultural Economics 78(May): 416-27.

Bartolucci, F., and A. Forcina. 2000. "A Likelihood Ratio Test for $\mathrm{MTP}_{2}$ within Binary Variables.” Annals of Statistics 28(4): 1206-18.

Beach, C. M., and R. Davidson. 1983. "Distribution-Free Statistical Inference with Lorenz Curves and Income Shares.” Review of Economic Studies 50(October): 723-35.

Beach, C. M., and J. Richmond. 1985. "Joint Confidence Intervals for Income Shares and Lorenz Curves." International Economic Review 26(June): 439-50.

Chambers, R. G. 1989. "Insurability and Moral Hazard in Agricultural Insurance Markets.” American Journal of Agricultural Economics 71(August): 604-16.

Chow, K. V. 1989. "Statistical Inference for Stochastic Dominance: A Distribution Free Approach.” PhD thesis, Department of Finance, University of Alabama.

Dardanoni, V., and A. Forcina. 1998. “A Unified Approach to Likelihood Inference on Stochastic Ordering in a Nonparametric Context." Journal of the American Statistical Association 93(September): 1112-23.

—. 1999. "Inference for Lorenz Curve Orderings.” Econometrics Journal 2(1): 49-75.

Davidson, R., and J-Y. Duclos. 2000. "Statistical Inference for Stochastic Dominance and for the Measurement of Poverty and Inequality.” Econometrica 68(November): 1435-64.

Duffy, M., and D. Smith. 2002. "Estimated Costs of Crop Production in Iowa-2002." University Extension, Iowa State University. January.

Dykstra, R., S. Kochar, and T. Robertson. 1995. "Inference for Likelihood Ratio Ordering in the TwoSample Problem." Journal of the American Statistical Association 90(September): 1034-40.

Greene, W. H. 2000. Econometric Analysis, 4th ed. Upper Saddle River, NJ: Prentice-Hall.

Hendricks, K., R. H. Porter, and C. A. Wilson. 1990. “Auctions for Oil and Gas Leases with An Informed Bidder and A Random Reservation Price." Discussion paper No. 910, Center for Mathematical Studies in Economics and Management Science, Northwestern University.

Holmström, B. 1979. "Moral Hazard and Observability.” The Bell Journal of Economics 10(Spring): 74-91. 
Innes, R. 1993. "Financial Contracting under Risk Neutrality, Limited Liability and Ex Ante Asymmetric Information." Economica 60(February): 27-40.

Iowa Department of Agriculture. various years. Iowa Agricultural Statistics. Annual publication, various years. Des Moines, IA.

Kaur, A., B. L. S. Prakasa Rao, and H. Singh. 1994. "Testing for Second-Order Stochastic Dominance of Two Distributions.” Econometric Theory 10(December): 849-66.

Klecan, L., R. McFadden, and D. McFadden. 1991. “A Robust Test for Stochastic Dominance.” Mimeo., Massachusetts Institute of Technology.

Koh, W. T. H. 1992. “A Note on Modelling Tournaments.” Journal of Economics 55(3): 297-308.

Kudô, A. 1963. "A Multivariate Analogue of the One-Sided Test.” Biometrika 50(December): 403-18.

Landsberger, M., and I. Meilijson. 1990. "Demand for Risky Financial Assets: A Portfolio Analysis." Journal of Economic Theory 50(February): 204-13.

List, J. A., and J. F. Shogren. 1999. "Price Information and Bidding Behavior in Repeated Second-Price Auctions." American Journal of Agricultural Economics 81(November): 942-49.

Milgrom, P. R. 1981a. "Good News and Bad News: Representation Theorems and Applications." The Bell Journal of Economics 12(Autumn): 380-91.

1981b. "Rational Expectations, Information Acquisition, and Competitive Bidding." Econometrica 49(July): 921-43.

Milgrom, P. R., and R. J. Weber. 1982. "A Theory of Auctions and Competitive Bidding." Econometrica 50(September): 1089-122.

Richmond, J. 1982. "A General Method for Constructing Simultaneous Confidence Intervals." Journal of the American Statistical Association 77(June): 455-60.

Robertson, T., F. T. Wright, and R. Dykstra. 1988. Order Restricted Statistical Inference. New York: John Wiley \& Sons.

Savin, N. E. 1984. "Multiple Hypothesis Testing." In Handbook of Econometrics, vol. 2. Edited by Z. Griliches and M. D. Intriligator. Amsterdam: Elsevier Science Publishers, pp. 828-79.

Shaked, M., and G. J. Shanthikumar. 1994. Stochastic Orders and Their Applications. San Diego: Academic Press.

Shogren, J. F., S. Y. Shin, D. J. Hayes, and J. B. Kliebenstein. 1994. "Resolving Differences in Willingness to Pay and Willingness to Accept." American Economic Review 84(March): 255-70.

Wolak, F. A. 1989. “Testing Inequality Constraint in Linear Econometric Models.” Journal of Econometrics 41(June): 205-35.

Zheng, B. 2002. "Testing Lorenz Curves with Non-Simple Random Samples." Econometrica 70(May): $1235-43$.

Zheng, B., and B. J. Cushing. 2001. "Statistical Inference for Testing Inequality Indices with Dependent Samples." Journal of Econometrics 101(April): 315-35. 